\title{
UTILIZAÇÃO DA SERRAGEM NA PRODUÇÃO DE COMPÓSITOS PLÁSTICO-MADEIRA
}

Fábio Minoru Yamaji ${ }^{1}$ Arnaud Bonduelle ${ }^{2}$

\section{RESUMO}

Esta pesquisa utilizou materiais reciclados para a produção de compósitos plástico-madeira ou WPC (woodplastic composites). A madeira utilizada na forma de pó e a serragem foram misturadas ao polietileno de baixa densidade - PEBD reciclado em uma extrusora monorrosca de $75 \mathrm{~mm}$ de diâmetro. Foram testadas formulações com $10 \%, 20 \%, 40 \%$ e $50 \%$ (em peso) de madeira. O teor de umidade inicial da madeira foi de $5,16 \%$ para o pó e $7,32 \%$ para a serragem. $\mathrm{O}$ conjunto de temperaturas da extrusora variou de $135^{\circ} \mathrm{C}$ a $150^{\circ} \mathrm{C}$. Os resultados mostraram que a produção dos compósitos foi possível para as formulações com até $20 \%$ de madeira.

Palavras-chave: compósitos plástico-madeira, WPC, resíduos de madeira, reciclagem

\section{USE OF SAWDUST IN WOOD-PLASTIC COMPOSITES PRODUCTION}

\begin{abstract}
This research aimed the use of recycled materials to produce WPC (wood-plastic composites). The wood was used as sawdust and wood flour and the plastic material used was the low density polyethylene- LDPE recycled. The $75 \mathrm{~mm}$ single screw extruder was used in the composite's production. Formulations with $10 \%, 20 \% 40 \%$ and $50 \%$ (in weight) of wood was tested. The wood humidity content was $5,16 \%$ for powder and $7,32 \%$ for sawdust. The extruder temperatures ranged from $135^{\circ} \mathrm{C}$ to $150^{\circ} \mathrm{C}$. The results showed that the composite production was possible for the formulations up to $20 \%$ wood content.

Keywords: wood-plastic composites, WPC, wood residues, recycle
\end{abstract}

\section{INTRODUÇÃO}

No setor madeireiro, a grande quantidade de resíduos gerados sempre foi motivo para preocupações. Dentre esses, o pó da lixa e a serragem merecem especial atenção por serem materiais de baixa densidade, exigindo maior espaço para a estocagem, além de serem materiais altamente explosivos. Atualmente, cada vez mais os resíduos vêm despertando o interesse de pesquisadores e empresários, principalmente para verificar as possibilidades de reutilização desses materiais.

Uma das alternativas para os resíduos da indústria madeireira é a utilização na produção de compósito plástico-madeira. Segundo Stark, White e Clemons (1997), o compósito plástico-madeira ou WPC (woodplastic composites) está se tornando o material mais importante dentro do processo da reciclagem.

Uma definição bem simples para o compósito plástico-madeira é dada por Koenig e Sypkens (2002). Segundo os autores, o compósito plástico-madeira é uma mistura de pó de madeira com algum tipo de resina plástica.

O uso dos compósitos plástico-madeira está crescendo rapidamente. Isso se deve às vantagens desse produto em relação à madeira, como não rachar, não empenar e exigir pouca ou nenhuma manutenção (Brandt e Fridley, 2003).

Para o futuro existe uma perspectiva de aumento para o uso do compósito plásticomadeira, pois, dependendo da formulação, pode-se obter produtos com propriedades superiores ao plástico e à própria madeira (Koenig e Sypkens, 2002).

O objetivo deste estudo foi produzir

\footnotetext{
fmyamaji@floresta.ufpr.br, doutorando em Engenharia Florestal, Universidade Federal do Paraná.

arnaud@floresta.ufpr.br, Dr., Professor do Programa de Pós-graduação Eng. Florestal - UFPR 
compósitos plástico-madeira ou WPC (woodplastic composites) utilizando resíduos de uma indústria madeireira. Os objetivos específicos foram:

a) verificar a possibilidade de se produzir esses compósitos em uma extrusora monorrosca;

b) verificar a possibilidade de se produzir esses compósitos sem nenhum tratamento prévio dos resíduos da madeira;

c) verificar a influência da granulometria dos resíduos da madeira no processamento dos compósitos plástico-madeira.

\section{REVISÃO BIBLIOGRÁFICA}

Os compósitos plástico-madeira ou WPC (wood plastic composites) começaram a ser produzido na Europa e estão sendo fabricado nos Estados Unidos há décadas. Entretanto, o maior crescimento vem ocorrendo a partir da última década (Clemons, 2002).

$\mathrm{Na}$ verdade, as primeiras pesquisas com o compósito plástico-madeira foram na década de 50 onde as indústrias automotivas começaram adicionar pó de madeira ao polipropileno (PP) na fabricação de algumas partes internas dos carros. No final da década de 80 o compósito plástico-madeira começou a ser usado na fabricação de decks (Koenig; Sypkens, 2002).

Atualmente os compósitos plásticomadeira já estão sendo utilizados na fabricação de uma série de produtos. As aplicações vão desde brinquedos até construções marinhas Youngquist (1995), British Plastic \& Rubber (2001) e Koenig e Sypkens (2002).

Nos compósitos plástico-madeira, o plástico é o material usado para melhorar as características como a resistência à umidade, ao ataque de insetos e fungos (Schut, 1999). Entretanto, de acordo com English (2002) numa temperatura onde muitos plásticos são processados, a madeira irá se degradar. Por isso, as formulações dos compósitos plásticomadeira ficam restritas a determinados tipos de plásticos onde a temperatura de processamento é relativamente baixa, como o polietileno (PE) e o polipropileno (PP). Além disso, esses polímeros apresentam baixo custo e têm boas propriedades mecânicas para peças não estruturais.
Os reforços tipicamente usados nos plásticos, como a fibra de vidro e outros minerais, são materiais caros e pesados. As fibras de madeira, que são mais baratas e mais leves, também podem ser utilizadas como reforço para os plásticos. Tratando-se de compósitos plástico-madeira, tanto o plástico quanto a madeira podem ser obtidos a partir de reciclados (English, 2002).

Youngquist (1995), Joseph et al. (1996), Mattoso (1999), Joseph et al. (2002) e Colom et al. (2003) citam que as vantagens na utilização de fibras vegetais para compósitos poliméricos são a melhoria do desempenho mecânico de plásticos convencionais, a diminuição do impacto ambiental, a reciclabilidade e o menor custo.

A madeira tem ainda a função de aumentar a rigidez dos compósitos, melhorar as propriedades de usinabilidade além de ter um custo menor que a resina (Schut, 1999).

De acordo com Stark (1999), Stark e Rowlands (2003) o pó de madeira é a forma mais comum encontrada nas misturas com termoplásticos.

A madeira e o plástico podem ser combinados usando a tecnologia da mistura por aquecimento e fusão. A produção dos compósitos plástico-madeira normalmente envolve o processo de extrusão. As matériasprimas (plástico e madeira) são misturados em uma extrusora, formando os granulados (Yougquist, 1995; Clemons, 2002).

Para Saheb e Jog (1999), o processamento de compósitos plástico-madeira envolve a extrusão dos componentees a uma determinada temperatura de fusão seguida por uma operação de injeção para dar a forma aos objetos.

A vantagem do compósito plásticomadeira é sua capacidade de ser processada como o plástico. $\mathrm{O}$ material pode ser injetado e produzir peças sem a necessidade de novas operações. O compósito também pode ser transformado em lâminas e moldados como os plásticos (Koenig; Sypkens, 2002).

Saheb e Jog (1999), Koenig e Sypkens (2002) lembram que as características dos compósitos plástico-madeira são influenciadas pelo método de processamento.

Koenig e Sypkens (2002) apresentam alguns pontos básicos para o processamento dos compósitos plástico-madeira: 
a) sempre manter as temperaturas do processo o mais baixo possível. Temperaturas abaixo de $200^{\circ} \mathrm{C}$ são recomendadas para evitar a degradação da madeira. Sempre permitir adequada saída de gases. A umidade resultante do processo de extrusão precisa ter uma saída da extrusora;

b) quanto mais uniforme a mistura, menor poderá ser a temperatura do processo;

c) extrusoras com rosca dupla são mais adequadas à mistura do que extrusoras com rosca simples.

Koenig e Sypkens (2002) afirmam que as pesquisas com os compósitos plásticomadeira estão buscando formulações para conseguir cada vez mais uma maior proximidade estética com a madeira.

De acordo com Stark (2001), os compósitos plástico-madeira estão sendo examinados também para aplicações estruturais e em usos externos em condições adversas.

Devido à incompatibilidade da madeira, material polar, com as poliolefinas (PP, PE), materiais apolares, é necessário promover a adesão na interface plástico-madeira no compósito para que o produto apresente boas propriedades mecânicas. Em geral é utilizado um compatibilizante polimérico para fazer a modificação superficial da madeira (fibra) ou da matriz plástica para efetivamente haver um reforço da matriz polimérica.

Conforme avança as pesquisas, melhora a performance dos compósitos plásticomadeira. No futuro deverá ser desenvolvido um produto que atenda a funções estruturais (English, 2002).

\section{METODOLOGIA}

\section{Materiais}

As matérias-primas utilizadas neste estudo para a produção de compósitos plásticomadeira foram madeira e plástico reciclados. Não foi empregado nenhum tipo de aditivo.

A madeira utilizada nos testes foi os resíduos gerados por uma indústria de compensados localizada na região de CuritibaPR. A indústria processa apenas folhosas oriundas da região Norte do Brasil. As espécies mais utilizadas são a copaíba (Copaifera sp) e a amescla (Protium heptaphyllum). A densidade das espécies varia entre 0,60 e $0,7 \mathrm{~g} / \mathrm{cm} 3$. Os resíduos utilizados foram o pó da lixadeira calibradora e a serragem da esquadrejadeira. Esses materiais já passaram por um processo de produção e apresentam um teor de umidade menor que $8 \%$, que pode ser considerado um valor baixo para a madeira. Assim sendo, os resíduos foram utilizados na produção dos compósitos sem nenhum processo de secagem.

O polímero utilizado foi o polietileno de baixa densidade (PEBD) reciclado. O material foi fornecido pela Indústria Roguiplast. A empresa trabalha com reciclagem e produção de tubos e mangueiras de polietileno de baixa densidade (PEBD).

Os testes para a produção dos compósitos plástico-madeira foram realizados nas Indústrias Roguiplast, localizada no município de Bauru-SP. Para o processamento foram utilizados os equipamentos convencionais de uma recuperadora de plástico, sem nenhum tipo de modificação nos equipamentos.

Embora Koenig e Sypkens (2002) recomendem o uso da extrusora de rosca dupla para o processamento dos compósitos plásticomadeira, a extrusora utilizada neste estudo foi do tipo monorrosca, por ser o equipamento mais comumente encontrado nas indústrias de plástico. A extrusora utilizada foi to tipo monorrosca de $75 \mathrm{~mm}$, sem sistema de degasagem, com cinco zonas de aquecimento, saída de 7 fios e corte via espaguete.

Antes de se iniciar o processo da produção foi feita a análise da madeira para a caracterização do material quanto ao tamanho das partículas e o seu teor de umidade. A partir dos materiais, serragem e pó da lixadeira, coletados na indústria foram retirados amostras para as análises granulométricas bem como para a determinação da densidade e do teor de umidade dos materiais. As análises foram conduzidas nos laboratórios da UFPR.

Para a análise granulométrica foi utilizado o equipamento denominado granuteste, com um conjunto de 4 peneiras (20, 40, 60 e 100 mesh).

Embora a densidade da madeira, na forma sólida (tora, tábua, viga, etc.) seja conhecida, é importante também saber a quantidade de madeira existente num determinado volume de serragem. Segundo Kollmann e Côté (1984), é essencial para as 
indústrias ter um conhecimento do peso médio por unidade de volume para os diferentes tipos de resíduos da madeira.

A determinação da densidade desses materiais (pó e serragem) foi feita através da relação peso/volume utilizando-se um copo de Becker.

$\mathrm{O}$ teor de umidade foi determinado colocando-se o material em estufa a $102^{\circ} \mathrm{C} \pm 2$ até peso constante.

O polímero utilizado nos testes para a produção dos compósitos plástico-madeira foi o mesmo polietileno de baixa densidade
(PEBD) reciclado, normalmente utilizado pela indústria na linha de produção. Para $o$ polímero também foram coletadas amostras para determinar o teor de umidade (antes da mistura com a madeira).

\section{Misturas}

Para a produção dos compósitos plástico-madeira foram programadas 4 formulações para cada um dos dois tipos de resíduo (pó e serragem), resultando num total de 8 formulações (ver tabela 1).

Tabela 1: Formulações programadas

Table 1: Programmed formulations

\begin{tabular}{ccccc}
\hline Formulações & \multicolumn{4}{c}{$\%$ em peso } \\
\cline { 2 - 5 } & Pó (lixadeira) & + PEBD & Serragem & + PEBD \\
1 & 10 & 90 & 10 & 90 \\
2 & 20 & 80 & 20 & 80 \\
3 & 40 & 60 & 40 & 60 \\
4 & 50 & 50 & 50 & 50 \\
\hline
\end{tabular}

PEBD=polietileno de baixa densidade

Cada formulação (madeira + PEBD) foi preparada e misturada previamente para, então, ser colocada na extrusora. O ajuste da extrusora para processar os compósitos plástico-madeira foi feito nas mesmas condições utilizadas pela empresa para processar o PEBD (polietileno de baixa densidade) sem nenhum tipo de mistura (100\% plástico). $\mathrm{O}$ ajuste das temperaturas nas cinco zonas de aquecimento ficou entre $135^{\circ} \mathrm{C}$ e $150^{\circ} \mathrm{C}$. A temperatura da água para resfriamento do espaguete era de $20^{\circ} \mathrm{C}$.

\section{RESULTADOS E DISCUSSÃO}

\section{Granulometria}

De acordo com a tabela 2, observa-se que a serragem (resíduo da esquadrejadeira) apresentou uma granulometria que variou desde 20 mesh até 100 mesh, sendo que $70 \%$ do material esteve entre 40 e 100 mesh. O pó da lixa é um material mais fino e mais uniforme, quando comparado com a serragem. A granulometria variou entre 60 mesh e $>100$ mesh, sendo que $84 \%$ do pó apresentou uma granulometria $>100$ mesh. A distribuição da granulometria pode ser vista na figura 1 .

Tabela 2: Resultados da análise granulométrica

Table2: Results of the granulometric analysis

\begin{tabular}{lcc}
\hline Mesh & \% de material retido na peneira \\
\hline & Serragem & Pó da lixa \\
20 & 3,0 & 0 \\
40 & 18,0 & 0 \\
60 & 30 & 2,0 \\
100 & 22 & 14,0 \\
$>100$ & 27 & 84,0 \\
\hline
\end{tabular}


Utilização da serragem na produção...

Figura 1: Histograma de distribuição das partículas

Figure: Histogram of the particles distributions

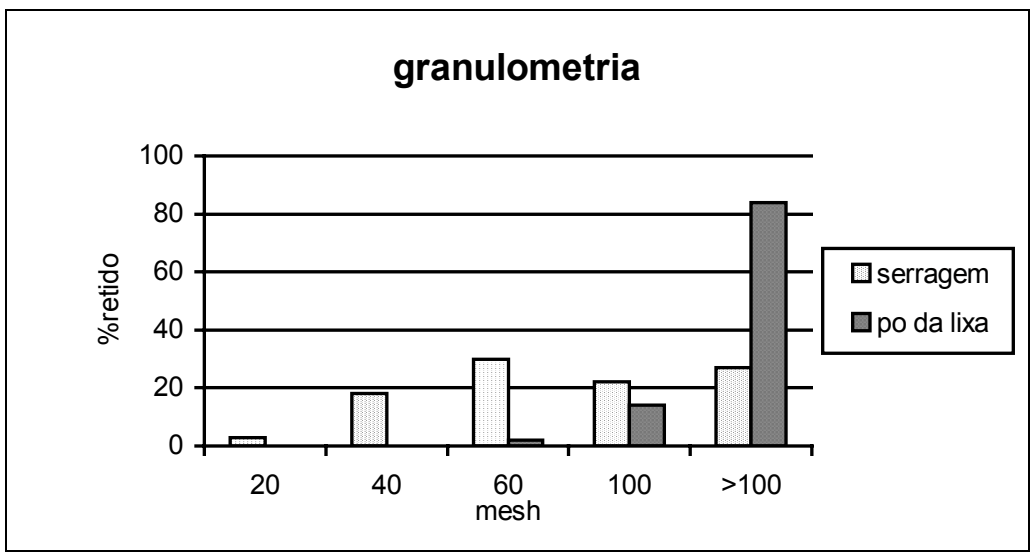

\section{Densidade}

$\mathrm{Na}$ tabela 3 são apresentados os resultados das densidades encontradas para os materiais utilizados na produção dos compósitos plástico-madeira. Observa-se que o pó da lixa, material que teve a granulometria mais fina (>100 mesh), é o material que apresenta a densidade mais baixa. De acordo com a REVISTA DO PLÁSTICO (2002), Koenig e Sypkens (2002), a baixa densidade dos resíduos da madeira é um dos fatores para que ocorram inconsistências no produto, como a separação de materiais, acarretando na produção de um compósito plástico-madeira de baixa qualidade.

Tabela 3: Resultados dos testes de densidade aparente

Table3: $\quad$ Results from the apparent density test

\begin{tabular}{lcccc}
\hline & Serragem & Pó da lixa & PEBD (granulado) \\
\hline $\begin{array}{l}\text { Densidade } \\
\left(\mathrm{g} / \mathrm{cm}^{2}\right)\end{array}$ & 0,25 & 0,14 & 0,32 \\
\hline
\end{tabular}

$\mathrm{PEBD}=$ polietileno de baixa densidade

\section{Teor de Umidade}

A tabela 4 apresenta os resultados dos testes de umidade dos materiais antes das misturas. Segundo Kollmann e Côté (1984), a umidade de equilíbrio de uma determinada espécie de madeira varia de acordo com a umidade relativa do ar e da histerese. Entretanto, considerando uma umidade relativa do ar entre $60 \%$ e $90 \%$, pode-se dizer que a umidade de equilíbrio da madeira deve ficar entre $10 \%$ e $18 \%$.
Assim sendo, o conteúdo de umidade encontrado de 7,32\% para a serragem e 5,16\% para o pó da lixadeira podem ser considerados baixos. Entretanto, de acordo com Schut (1999) e Clemons (2002), um teor de umidade acima de $1 \%$ pode ser considerado elevado para processamento em extrusoras.

Para o polietileno de baixa densidade (PEBD) foi encontrado um teor de umidade de $0,35 \%$. O material com esse conteúdo de umidade é normalmente utilizado pela indústria (reciclagem e produção de tubos).

Tabela 4: Resultados do teste de teor de umidade

Table 4: Results from the humidity content test

\begin{tabular}{cccc}
\hline & Serragem & Pó da lixa & PEBD (granulado) \\
\hline Teor de umidade & $7,32 \%$ & $5,16 \%$ & $0,35 \%$ \\
\hline
\end{tabular}

PEBD=polietileno de baixa densidade 


\section{Produção dos compósitos plástico-madeira}

A produção dos compósitos plásticomadeira foi dividida em lotes, de acordo com as formulações apresentadas na tabela 1 .

Em todos os processos de produção dos compósitos plástico-madeira houve a liberação de um odor característico diferente do cheiro verificado durante o processamento do polietileno - PEBD (100\%). Quanto maior o percentual de madeira na formulação dos compósitos processados, mais intenso foi percebido o odor, lembrando o cheiro de madeira queimada.

Outra característica observada foi a geração de gases em todos os processos. Durante o processo de fusão do plástico $\left(135 \sim 150^{\circ} \mathrm{C}\right)$, toda umidade contida na mistura transforma-se em vapor. Esses gases são eliminados juntamente com o material. E, quanto maior o volume de madeira nas formulações, maior o teor de umidade na mistura (ver tabela 5). A produção dos compósitos plástico-madeira foi diretamente afetada pelo volume de gases gerados no interior da extrusora.

As duas formulações com $10 \%$ apresentaram um teor de umidade da mistura de $0,8 \%$ e $1,0 \%$ para o pó e para a serragem respectivamente (ver TABELA 5). Esses índices estão dentro dos limites citados por Schut (1999) e Clemons (2002) (ver 4.3 TEOR DE UMIDADE). A produção de gases pôde ser observada, mas não chegou a interferir negativamente na produção.

De acordo com a tabela 5, as formulações com $20 \%$ tiveram um conteúdo de umidade de $1,3 \%$ e $1,7 \%$ para o pó e para a serragem respectivamente. Embora esses índices estejam acima do teor recomendado, a produção da formulação com o pó (lixadeira) não foi tão afetada. Já a produção da formulação com a serragem $(1,7 \%$ de umidade na mistura) sofreu algumas interrupções durante o processo.

As formulações com $40 \%$ de madeira resultaram num teor de umidade da mistura de $3,1 \%$ para a formulação com a serragem e de $2,3 \%$ para a formulação que utilizou o pó da lixadeira. Ambas as formulações com 40\% tiveram sua produção prejudicada pela formação de gases. O fluxo dos espaguetes foi constantemente interrompido para liberação dos gases que era formado no interior da extrusora.

Tabela 5: Conteúdo de umidade das misturas

Table5: $\quad$ Content of humidity of the mixtures

\begin{tabular}{ccc}
\hline Formulações & \multicolumn{2}{c}{ Teor de umidade (\%) } \\
\cline { 2 - 3 } (\% de madeira) & Serragem & Pó (lixadeira) \\
10 & 1,0 & 0,8 \\
20 & 1,7 & 1,3 \\
40 & 3,1 & 2,3 \\
50 & 3,8 & 2,8 \\
\hline
\end{tabular}

$\mathrm{Na}$ fase de preparação dos compósitos (pesagem e mistura), houve maior dificuldade para se trabalhar com o pó da lixa por ser um material muito fino. Entretanto, durante a fase de extrusão não foi possível observar a influência da granulometria dos materiais no processo de produção dos compósitos plásticomadeira.

As duas formulações com $50 \%$, tanto para o pó quanto a serragem, não foram produzidas, haja vista que as formulações com $40 \%$ já apresentaram restrições (geração de gases) durante seu processamento na extrusora.

\section{CONCLUSÃO}

Os testes mostraram que é possível a produção de compósitos plástico-madeira a partir de materiais reciclados.

Considerando que a indústria utiliza o PEBD (sem a madeira) nos processos de reciclagem sem alteração na sua produção, as dificuldades observadas durante 0 processamento dos compósitos plásticomadeira podem ser atribuídas à madeira.

O pó da lixa é um material mais difícil para se manusear (por apresentar uma granulometria muito fina) do que a serragem. 
As duas granulometrias testadas (pó e serragem) apresentaram o mesmo comportamento durante a produção (extrusora).

Foi observado e, confirmado que a umidade é um fator determinante na produção de compósitos plástico-madeira. A produção dos compósitos sem um pré-tratamento da madeira limitou a produção a formulações com um conteúdo máximo de $20 \%$ (em peso) de madeira.

Mesmo utilizando os equipamentos convencionais existentes numa indústria recuperadora de plástico, sem modificações, a produção de compósitos plástico-madeira foi possível. Entretanto, a extrusora monorrosca, utilizada neste estudo, apresentou limitações quanto a liberação dos gases. Sem um sistema de degasagem na extrusora, a produção de compósitos plástico-madeira ficou muito limitada e restrita a determinadas formulações.

\section{RECOMENDAÇÕES}

Este estudo procurou demonstrar a possibilidade da produção de compósitos plástico-madeira a partir de materiais reciclados. Entretanto, face às limitações observadas, é importante que novos estudos sejam conduzidos, sendo recomendado:

a) a produção dos compósitos em uma extrusora com sistema de degasagem a vácuo;

b) testar a produção com os resíduos (madeira) a um teor de umidade o mais baixo possível, ou seja, próximo a $0 \%$;

c) testar a produção de compósitos com o polímero virgem;

d) fazer testes físicos para analisar a qualidade do compósito produzido;

e) avaliar as propriedades mecânicas dos compósitos;

f) fazer uma compatibilização dos compósitos através da adição de um compatibilizante polimérico;

g) avaliar a mistura e a interface através da análise da fratura dos corpos de prova tracionados.

\section{REFERÊNCIAS}

BRANDT, C. W.; FRIDLEY, K. J. Effect of load rate on flexural properties of wood-plastic composites. Wood and Fiber Science, v.35, n. 1,p.135-147, 2003.

BRISTSH PLASTICS \& RUBBER. Growth in consumption of extruded wood-plastic composites. London: MCM Publishing Ltd., v.May, p.26, 2001.

CLEMONS, C. Wood-plastic composites in the United States. Forest Products Journal, v.52, n.6, p.10-18, 2002.

COLOM, X.; CAARRASCO, F.; PAGÈS, P.; CAÑAVATE, J. Effects of different treatments on the interface of HDPE/lignocellulosic fiber composites. Composites Science and Technology, v.63, p.161-69, 2003.

ENGLISH, B. Wood-filled plastics: an introduction. In: Annual meeting of Window and Door Manufacturers Association, 2000, USA. Summaries... Arizona, 2000.

JOSEPH, K.; VARGHESE, S.; KALAPRASAD, G.; THOMAS, S.; PRASANNAKUMARI, L.; KOSHY, P.; PAVITHRAN, C. Influence of interfacial adhesion on the mechanical properties and fracture behaviour of short sisal fibre reinforced polymer composites. European Polymer Journal, v.32, n.10, p.1243-50, 1996.

JOSEPH, P. V.; RABELLO, M. S.; MATTOSO, L. H. C.; JOSEPH, K.; THOMAS, S. Environmental effects on the degradation behaviour of sisal fibre reinforced polypropylene composites. Composites Science and Technology, v.62, p.1357-72, 2002.

KOENIG, K. M.; SYPKENS, C. W. Woodplastic composites for market share. Wood and Wood Products, v.107, n.5, p.49-58, 2002.

KOLlMANN, F. F. P.; CÔTÉ, W. A. Principles of wood science and technology. New York: Spring-Verlag, 1984 (reprint).

MATTOSO, L. H. C. Conferência internacional de compósitos reforçados com fibras vegetais. Polímeros: Ciência e Tecnologia, v.IX, n.2, p.16, 1999.

REVISTA DO PLÁSTICO Chicago mostra os rumos do plástico. Disponível em: 
http://www.plastico.com.br/revista/pm310/npe 20006.htm. Acesso em 04 jun. 2002.

SAHEB, D. N.; JOG, J. P. Natural fiber polymer composites: a review. Advances in Polymer Technology, v.18, n.4, p.351-63, 1999.

SCHUT, J. H. For compounding, sheet \& profile, wood is good. Plastics Technology, v.45, n.3, p.46(7), 1999.

STARK, N. Influence of moisture absorption on mechanical properties of wood flourpolypropylene composites. Journal of Thermoplastic Composite Materials, v.14, n.5, p.421-432, 2001.

STARK, N. M. Wood fiber derived from scrap pallets used in polypropylene composites. Forest Products Journal, v.49, n.6, p.39-46, 1999.
STARK, N. M.; ROWLANDS, R. E. Effects of wood fiber characteristics on mechanical properties of wood/polypropylene composites. Wood and Fiber Science, v.35, n.2, p.167-74, 2003.

STARK, N. M.; WHITE, R. H.; CLEMONS, C. M. Heat release rate of wood-plastic composites. Sampe Journal, v.33, n.5, p.2631, 1997.

YOUNGQUIST, J. A. The marriage of wood and nonwood materials. Forest Products Journal, v.45, n.10, p.25-30, 1995. 